\section{AB1388-HPR TREATMENT ADHERENCE AMONG PATIENTS WITH INFLAMMATORY ARTHRITIDES TREATED WITH BDMARDS: AN OBSERVATIONAL STUDY USING I-CQR5 QUESTIONNAIRE AND THE ADMINISTRATIVE CLAIMS DATABASE}

Letizia Moino ${ }^{1}$, Desirée Bastarolo ${ }^{1}$, Cristiana Visentin ${ }^{2}$, Francesca Ometto ${ }^{3}$, Ernesto De Menis ${ }^{4}$, Cecilia Giron ${ }^{5}$, Costantino Botsios ${ }^{3,4}$. ${ }^{1}$ LOCAL HEALTH UNIT 2, Hospital Pharmacy, Castelfranco Veneto -Montebelluna Hospitals, Castelfranco (TV), Italy, ${ }^{2}$ Local Health Unit 2, Planning and Management Control Department, Treviso, Italy; ${ }^{3}$ UNIVERSITY OF PADOVA, Department of Medicine, DIMED, PADOVA, Italy; ${ }^{4}$ Local Health Unit 2, Department of Clinical Medicine, Castelfranco Veneto -Montebelluna Hospitals, Montebelluna (TV), Italy, ${ }^{5}$ University of Padova, Department of Pharmaceutical andPharmacological Sciences, DSF, Padova, Italy

Background: Effective treatments have been introduced for inflammatory arthritides (IAs) in the last decades. However, adherence remains suboptimal. Objectives: The aim of the study was to assess treatment adherence among patients with IAs treated with bDMARDs in a community hospita with the Italian 5-item Compliance Questionnaire for Rheumatology (ICQR5) [1] and the hospital administrative claims database.

Methods: We conducted a monocentric, cross-sectional, observational study in IA patients (disease duration $>1$ year, undergoing treatment with self-administered bDMARDs, capable to complete the questionnaire unaided) recruited in a community hospital from February to October 2018. Treatment adherence was defined according to I-CQR5 and to the Medication Possession Ratio (MPR) obtained from the claims database. To investigate variables associated with adherence, demographic, social, and clinical characteristics were considered. I-CQR5 has 5 questions, with Likert-answering scale ranging from 1 to 4 , an algorithm allows to classify the patient as poorly or highly adherent (i.e. likely to take $>=80 \%$ of their medications correctly) [1]. MPR was obtained by dividing the number of dosage units withdrawn by patients by the prescribed number of dosage units. Agreement between the I-CQR5 and MPR definition of high adherence definitions was tested with McNemars test, while the association with patients characteristics with chi-square or Fishers exact test.

Results: A total 174 patients completed the I-CQR5 (median age 60 years, range $51-67 ; 37 \%$ males), affected by ankylosing spondylitis (16\%), rheumatoid arthritis (RA) (62\%), psoriatic arthritis (22\%) with a median duration of 15 years (range 10-20). Etanercept (49\%), adalimumab (20\%), abatacept $(8 \%)$ and tocilizumab $(7.5 \%)$ were the most prescribed first-line biologics. Association with a conventional synthetic DMARDs was prescribed to $3.4 \%$ of subjects. High adherence rate, assessed with I-CQR5, was $83 \%$ overall, and $85 \%$ in RA patients. Significant differences were observed between highly and poorly adherent patients according to ICQR5: lower educational status (lower primary or secondary school, and the use of cDMARDs were associated with poor adherence (both with $\mathrm{p}<0.001$ ); while the use of bDMARDs was negatively associated with high adherence $(p<0.05)$. High adherence rate measured with MPR was $72 \%$. The agreement between high adherence, measured with I-CQR5 and MPR, was low $\mathrm{p}=0.005$ )

Conclusion: The study showed that IA patients treated with bDMARDs with a higher educational level are more adherent to physician prescription, as assessed by I-CQR5. I-CQR5 might overestimate adherence compared to MPR. In our study, the high adherence rate measured with ICQR5 was higher compared with a report from a large outpatient clinic in the same area, which was limited to RA patients ( $85 \%$ vs $40,1 \%$ ) [1] Our study may suggest that adherence benefits more from a close and stable relationship with the health care practitioner, such as in our centre, which is smaller than large outpatients clinics in which patients are followed by a health care professionals equipe rather than a single physician.

\section{REFERENCES}

[1] Ometto F, et al. Ann Rheum Dis 2018;77(Suppl): A1322.

\section{AB1389-HPR THE ASSOCIATION BETWEEN THE AMOUNT OF DIASTASIS RECTI ABDOMINIS AND THE STRENGTH OF ABDOMINAL MUSCLES IN PREGNANT WOMEN}

Gülbala Nakip $^{1}$, Türkan Akbayrak ${ }^{1}$, Gamze Nalan Çinar ${ }^{1}$, Esra Üzelpasacı ${ }^{1}$, Emine Baran ${ }^{1}$, Ceren Orhan ${ }^{1}$, Serap Özgül ${ }^{1}$, Mehmet Sinan Beksaç ${ }^{2} .{ }^{1}$ Hacettepe University, Faculty of Health Sciences, Department of Physiotherapy and Rehabilitation, Ankara, Turkey; ${ }^{2}$ Hacettepe University, Faculty of Medicine, Department of Obstetrics and Gynecology, Ankara, Turkey

Background: Diastasis recti abdominis (DRA) is a common musculoskeletal problem, which is defined as a separation of the inter-recti distance between the two bellies of the rectus abdominis muscle at the linea alba. $^{1}$ Due to the hormonal, postural and musculoskeletal changes, and mechanical strain, the anterior abdominal wall becomes stretched and elongated during pregnancy. Studies have shown that DRA may occur between $27 \%$ and $100 \%$ in the second and third trimesters of pregnancy ${ }^{2}$. Although it has been reported that imbalance in the strength of the abdominal wall muscles altered with the facial tension, the role of abdominal muscle strength on DRA has not been clarified yet.

Objectives: The present study assessed the relation between the severity/amount of DRA and the degree of abdominal muscle (rectus abdominis (RA), external and internal oblique muscles) strength in pregnant women.

Methods: A total of 153 pregnant women between 14 and 35 weeks of a singleton pregnancy were included in the present study. The finger width method was used to measure the amount of DRA. The amount of separation was determined by the palpation of the medial sides of RA at three reference points: on the umbilicus, $4.5 \mathrm{~cm}$ above and $4.5 \mathrm{~cm}$ below of the umbilicus. After the subject contracts RA in hook lying position when her arms were in extended position, the size of the diastasis was measured by the number of finger. $^{3}$ The strength of abdominal muscles was assessed by the manual muscle test in supine hook lying position. The correlation between the amount of DRA and the degree of abdominal muscle strength was analyzed by the Spearmans correlation. Results: The mean age was $28.40 \pm 3.69$ years, mean Body Mass Index was $27.01 \pm 1.26 \mathrm{~kg} / \mathrm{m}^{2}$, and mean gestational age was $28.12 \pm 5.03$ weeks A negative correlation was found between the amount of the diastasis at $4.5 \mathrm{~cm}$ below of the umbilicus and the strength of the RA $(r=0.219$, $\mathrm{p}=0.007)$, right $(\mathrm{r}=0.296, \mathrm{p}=0.015)$ and left external oblique muscles $(r=0.293, p=0.017)$

Conclusion: Based on the findings of the present study, the separation of RA on the lower level of umbilicus may be negatively associated with the strength of RA and oblique abdominal muscles. Therefore, to decrease the severity of DRA, abdominal strengthening programs should be provided to pregnant women.

\section{REFERENCES}

[1] Van de Water, A. T. M., \& Benjamin, D. R. (2016). Measurement methods to assess diastasis of the rectus abdominis muscle (DRAM): a systematic review of their measurement properties and meta-analytic reliability generalisation. Manual therapy, 21, 41-53.

[2] B, K., Hilde, G., Tennfjord, M. K., Sperstad, J. B., \& Engh, M. E. (2017) Pelvic floor muscle function, pelvic floor dysfunction and diastasis recti abdominis: prospective cohort study. Neurourology and urodynamics, 36 (3), 716-721.

[3] Michalska, A., Rokita, W., Wolder, D., Pogorzelska, J., \& Kaczmarczyk, K. (2018). Diastasis recti abdominisa review of treatment methods. Ginekologia polska, 89(2), 97-101.

Disclosure of Interests: None declared DOI: 10.1136/annrheumdis-2019-eular.6975 